\title{
Parsec-scale Herbig-Haro outflows from intermediate mass stars
}

\author{
F. McGroarty ${ }^{1}$, T. P. Ray ${ }^{1}$, and J. Bally ${ }^{2}$ \\ 1 Dublin Institute for Advanced Studies, 5 Merrion Square, Dublin 2, Ireland \\ 2 Department of Astrophysical and Planetary Sciences and Center for Astrophysics and Space Astronomy, \\ University of Colorado, Campus Box 389, Boulder, CO 80309-0389, USA
}

Received 15 August 2003 / Accepted 28 October 2003

\begin{abstract}
While there are many parsec-scale Herbig-Haro (HH) outflows known to be driven by low-mass young stars, few are associated with their intermediate mass counterparts. Here we present the discovery of five such bipolar outflows. Of these, $\mathrm{LkH} \alpha 198,1548 \mathrm{C} 27$ IRS 1, LkH $\alpha 233$ and $\mathrm{LkH} \alpha 234$ were previously known to possess small-scale HH flows, while no such activity was observed before near IRAS $19395+2313$. The largest of the newly discovered outflows are seen in the vicinity of $\mathrm{LkH} \alpha 234$ and 1548C27 IRS 1, and stretch (in projection) $8 \mathrm{pc}$ and $7.5 \mathrm{pc}$ respectively. $\mathrm{LkH} \alpha 233$ which was previously known to power a spectroscopically detected small-scale $\left(\leq 10^{\prime \prime}\right)$ jet is now seen to drive a 3 pc outflow and $\mathrm{LkH} \alpha 198$ is shown here to power a 2 pc outflow. Two HH objects in the vicinity of IRAS 19395+2313 lead us to suggest that it may also be responsible for a 5 pc outflow. In total, 27 new HH objects/complexes were discovered. Examination of these parsec-scale outflows show that they have similar lengths, morphologies, and dynamical timescales as those from low-mass sources. Many appear to have blown out of the parent cloud, suggesting that their total lengths are much greater than optically observed. The degree of collimation of these outflows is similar to those from low-mass sources suggesting that the transition to more poorly-collimated outflows must occur at higher masses than the sources observed here.
\end{abstract}

Key words. ISM: Herbig-Haro objects - stars: formation - stars: individual: LkH $\alpha$ 198, 1548C27 IRS1, LkH $\alpha 233$, LkH $\alpha 234$

\section{Introduction}

HH objects are the shock-excited nebulous tracers of outflows from pre-main sequence stars. In many cases these outflows are collimated in the form of highly supersonic jets the existence of which appear to be intrinsicly linked to accretion by the underlying young stellar object (YSO) (Hartigan et al. 1995). Most known optical jets have low mass $\left(\sim 1 M_{\odot}\right)$ sources: either the embedded (IRAS Class I) counterparts of classical TTauri stars or classical TTauri stars themselves (Reipurth et al. 1997).

Turning to higher mass YSOs $\left(>10 M_{\odot}\right)$, such as those driving the Orion OMC 1 or Cepheus A outflows, one sees a very different picture (Allen \& Burton 1993; O'Dell et al. 1997; Hartigan et al. 2000). Their outflows, although highly energetic, often appear poorly collimated and more chaotic (Reipurth \& Bally 2001) than their low mass counterparts. This transformation begs two obvious inter-related questions: at what point does the transition occur and is it a smooth function of mass? To answer these questions one must examine outflows from YSOs of mass greater than $\sim 2 M_{\odot}$.

Optical outflows have been observed from a number of intermediate-mass $\left(2 M_{\odot} \leq \mathrm{M}_{*} \leq 10 M_{\odot}\right)$ YSOs, for example R Mon, LkH $\alpha$ 234, and AFGL 4029 (Ray et al. 1990 and

Send offprint requests to: F. McGroarty, e-mail: fmcg@cp.dias.ie references therein). Such stars, where optically visible, are known as Herbig Ae/Be stars (HAEBES) although their embedded counterparts have also been seen. However optical outflows from these YSOs are rare and there are a number of reasons for this. The initial mass function favours the production of low mass stars and therefore intermediate mass YSOs tend to be found at relatively large distances. More massive stars also have a faster evolutionary timescale - i.e. they evolve more quickly than low-mass YSOs and so their outflow phase is shorter. This would also make their outflows more difficult to detect. Another contributing factor could be that massive stars tend to be more obscured: intermediate and massive stars tend to be surrounded by large amounts of circumstellar gas and dust, making it harder to find an outflow at visual wavelengths. Finally there may well have been a historical bias towards studying outflows from low-mass stars. The situation however has changed in recent years as more and more studies focus on higher mass YSOs.

With these ideas in mind, we have investigated the occurrence of large-scale outflows from intermediate mass stars. By large scale we mean those stretching several parsecs e.g. the PV Cephei outflow at $2.6 \mathrm{pc}$ (Gomez et al. 1997; Reipurth et al. 1997), the HH 80/81 5.3 pc outflow (Marti et al. 1993) and the HH 354 outflow at 2.4 pc (Reipurth et al. 1997). We emphasise that these outflows have vastly longer associated timescales 
than those of "traditionally" observed flows. A small-scale $\mathrm{HH}$ jet close to its source has a dynamical timescale of only a few hundred years, whereas the HH objects in these parsecscale flows trace mass ejection over tens of thousands of years. They are, in effect, fossil records of the mass-loss histories of their parent star.

Newly detected parsec-scale outflows around five intermediate-mass young stars are discussed here (see also Tables 1 and 2). Of these, $\mathrm{LkH} \alpha$ 198, $\mathrm{LkH} \alpha 233$ and $\mathrm{LkH} \alpha 234$ are of spectral type A and $1548 \mathrm{C} 27$ is A7-F0. All of these stars were known to possess small-scale optical outflows. The one optically invisible YSO in our sample, IRAS 19395+2313, was not previously known to drive any outflow.

Section 2 describes how we made our observations and in Sect. 3 we present our newly discovered large-scale flows. The implications of our findings are discussed in Sect. 4 and our conclusions are drawn in Sect. 5.

\section{Observations}

To carry out our survey we used the Wide Field Camera (WFC) at the prime focus of the $2.5 \mathrm{~m}$ Isaac Newton Telescope at El Observatorio del Roque de los Muchachos (La Palma, Canary Islands). The WFC consists of four thin-coated EEV CCDs each with $2048 \times 410015 \mu \mathrm{m}^{2}$ pixels. One pixel projects to 0.33 on the sky. Three of the CCDs are positioned from north to south with their long axes adjoining. The fourth is attached to the west to form a square mosaic (34.2 wide) with its northwestern corner missing.

Our images were taken on nights between the 13th and the 21 st of July 1998 . Seeing was moderate at $1.15-1{ }^{\prime \prime} 5$ as measured from the images. $\mathrm{HH}$ objects were identified using a number of narrowband emission line filters: $\mathrm{H} \alpha\left(\lambda_{\mathrm{c}}=6568 \AA\right.$, $\Delta \lambda(F W H M)=95 \AA),[\mathrm{SII}]\left(\lambda_{\mathrm{c}}=6725 \AA, \Delta \lambda(F W H M)=80 \AA\right)$ and $[\mathrm{OIII}]\left(\lambda_{\mathrm{c}}=5008 \AA, \Delta \lambda(F W H M)=100 \AA\right)$. To distinguish HH emission from reflection nebulosity, we also took broadband images in $V$ and $I$. Exposure times for the narrowband and broadband images were typically 30 and $10 \mathrm{~min}$ respectively. The data was reduced using standard IRAF reduction procedures.

\section{Results for individual regions}

\section{1. $L k H \alpha 198 \&$ V376 Cas}

LkH $\alpha 198$ and its nearby companion, V376 Cas, are both Herbig Ae stars (Herbig 1960) located in the small dark cloud L1265, at a distance of 600 pc (Chavarria-K. 1985). An asymmetrical, bipolar molecular outflow in this region was noted by Canto et al. (1984). Strom et al. (1986) subsequently found the first optical outflow tracer, HH 161, a bright $\mathrm{HH}$ object some 12" from $\mathrm{LkH} \alpha 198$ at a position angle (PA) of $100^{\circ}$. Further observations by Goodrich (1993) yielded another HH knot $81^{\prime \prime}$ away at a PA of $153^{\circ}$. This object was rediscovered by Aspin \& Reipurth (2000) who refer to it as HH 461.

The discovery, however, of $\mathrm{LkH} \alpha 198 \mathrm{~B}$, a deeply embedded companion to $\operatorname{LkH} \alpha$ 198, by Lagage et al. (1993) raised
Table 1. Positions of the new HH objects found in this survey and their probable sources.

\begin{tabular}{|c|c|c|c|}
\hline Object & Source & $\alpha(\mathrm{J} 2000)$ & $\delta(\mathrm{J} 2000)$ \\
\hline HH 800 & UNKNOWN & $00^{\mathrm{h}} 11^{\mathrm{m}} 02.0^{\mathrm{s}}$ & $+58^{\circ} 55^{\prime} 04^{\prime \prime}$ \\
\hline НН 801 & $\mathrm{LkH} \alpha 198$ & $00^{\mathrm{h}} 11^{\mathrm{m}} 12.0^{\mathrm{s}}$ & $+58^{\circ} 54^{\prime} 01^{\prime \prime}$ \\
\hline HН 802 & $\mathrm{LkH} \alpha 198$ & $00^{\mathrm{h}} 11^{\mathrm{m}} 44.5^{\mathrm{s}}$ & $+58^{\circ} 42^{\prime} 39^{\prime \prime}$ \\
\hline HН 803 & 1548C27 IRS 1 & $19^{\mathrm{h}} 42^{\mathrm{m}} 47.0^{\mathrm{s}}$ & $+23^{\circ} 22^{\prime} 19^{\prime \prime}$ \\
\hline НH 804 & IRAS $19395+2313$ & $19^{\mathrm{h}} 42^{\mathrm{m}} 10.4^{\mathrm{s}}$ & $+23^{\circ} 21^{\prime} 49^{\prime \prime}$ \\
\hline НH 805 & IRAS $19395+2313$ & $19^{\mathrm{h}} 41^{\mathrm{m}} 41.5^{\mathrm{s}}$ & $+23^{\circ} 20^{\prime} 34^{\prime \prime}$ \\
\hline НH 806 & UNKNOWN & $19^{\mathrm{h}} 42^{\mathrm{m}} 03.4^{\mathrm{s}}$ & $+23^{\circ} 20^{\prime} 02^{\prime \prime}$ \\
\hline НH 807 & UNKNOWN & $19^{\mathrm{h}} 42^{\mathrm{m}} 07.1^{\mathrm{s}}$ & $+23^{\circ} 19^{\prime} 54^{\prime \prime}$ \\
\hline НН 808 & $\mathrm{LkH} \alpha 233$ & $22^{\mathrm{h}} 34^{\mathrm{m}} 35.6^{\mathrm{s}}$ & $+40^{\circ} 39^{\prime} 42^{\prime \prime}$ \\
\hline НН 809 & $\mathrm{LkH} \alpha 233$ & $22^{\mathrm{h}} 34^{\mathrm{m}} 30.4^{\mathrm{s}}$ & $+40^{\circ} 39^{\prime} 01^{\prime \prime}$ \\
\hline НH 810 & $\mathrm{LkH} \alpha 233$ & $22^{\mathrm{h}} 34^{\mathrm{m}} 21.2^{\mathrm{s}}$ & $+40^{\circ} 37^{\prime} 34^{\prime \prime}$ \\
\hline НH 811 & $\mathrm{LkH} \alpha 233$ & $22^{\mathrm{h}} 34^{\mathrm{m}} 14.5^{\mathrm{s}}$ & $+40^{\circ} 36^{\prime} 48^{\prime \prime}$ \\
\hline НH 812 & $\mathrm{LkH} \alpha 233$ & $22^{\mathrm{h}} 34^{\mathrm{m}} 11.6^{\mathrm{s}}$ & $+40^{\circ} 36^{\prime} 33^{\prime \prime}$ \\
\hline НН 813 & $\mathrm{LkH} \alpha 233$ & $22^{\mathrm{h}} 34^{\mathrm{m}} 06.6^{\mathrm{s}}$ & $+40^{\circ} 36^{\prime} 18^{\prime \prime}$ \\
\hline НH 814 & $\mathrm{LkH} \alpha 233$ & $22^{\mathrm{h}} 35^{\mathrm{m}} 01.4^{\mathrm{s}}$ & $+40^{\circ} 43^{\prime} 33^{\prime \prime}$ \\
\hline НН 815 & $\mathrm{LkH} \alpha 234$ & $21^{\mathrm{h}} 44^{\mathrm{m}} 29.9^{\mathrm{s}}$ & $+66^{\circ} 13^{\prime} 42^{\prime \prime}$ \\
\hline HН 816 & $\mathrm{LkH} \alpha 234$ & $21^{\mathrm{h}} 44^{\mathrm{m}} 26.4^{\mathrm{s}}$ & $+66^{\circ} 10^{\prime} 58^{\prime \prime}$ \\
\hline НH 817 & $\mathrm{LkH} \alpha 234$ & $21^{\mathrm{h}} 44^{\mathrm{m}} 13.3^{\mathrm{s}}$ & $+66^{\circ} 10^{\prime} 55^{\prime \prime}$ \\
\hline НH 818 & $\mathrm{LkH} \alpha 234$ & $21^{\mathrm{h}} 43^{\mathrm{m}} 57.7^{\mathrm{s}}$ & $+66^{\circ} 10^{\prime} 26^{\prime \prime}$ \\
\hline НН 819 & $\mathrm{LkH} \alpha 234$ & $21^{\mathrm{h}} 44^{\mathrm{m}} 01.0^{\mathrm{s}}$ & $+66^{\circ} 09^{\prime} 52^{\prime \prime}$ \\
\hline НH 820 & $\mathrm{LkH} \alpha 234$ & $21^{\mathrm{h}} 43^{\mathrm{m}} 47.9^{\mathrm{s}}$ & $+66^{\circ} 09^{\prime} 50^{\prime \prime}$ \\
\hline НH 821 & $\mathrm{LkH} \alpha 234$ & $21^{\mathrm{h}} 43^{\mathrm{m}} 43.4^{\mathrm{s}}$ & $+66^{\circ} 08^{\prime} 47^{\prime \prime}$ \\
\hline HH 103 A & $\mathrm{LkH} \alpha 234$ & $21^{\mathrm{h}} 42^{\mathrm{m}} 20.7^{\mathrm{s}}$ & $+66^{\circ} 03^{\prime} 31^{\prime \prime}$ \\
\hline НH 822 & $\mathrm{LkH} \alpha 234$ & $21^{\mathrm{h}} 41^{\mathrm{m}} 42.1^{\mathrm{s}}$ & $+66^{\circ} 01^{\prime} 45^{\prime \prime}$ \\
\hline HH 823 & UNKNOWN & $21^{\mathrm{h}} 43^{\mathrm{m}} 27.9^{\mathrm{s}}$ & $+66^{\circ} 11^{\prime} 46^{\prime \prime}$ \\
\hline НH 824 & IRAS $21416+6556$ & $21^{\mathrm{h}} 42^{\mathrm{m}} 56.9^{\mathrm{s}}$ & $+66^{\circ} 09^{\prime} 10^{\prime \prime}$ \\
\hline НH 825 & IRAS $21416+6556$ & $21^{\mathrm{h}} 42^{\mathrm{m}} 39.2^{\mathrm{s}}$ & $+66^{\circ} 10^{\prime} 56^{\prime \prime}$ \\
\hline
\end{tabular}

the question of which of these two stars is the primary outflow source in this region. In their study Corcoran et al. (1995) (hereafter referred to as CRB) concluded there are two separate outflows with their origin in the vicinity of $\mathrm{LkH} \alpha 198$ : one driven by $\mathrm{LkH} \alpha 198$ itself and the other by $\mathrm{LkH} \alpha 198 \mathrm{~B}$. Their observations of HH 161 revealed a tail pointing back towards $\mathrm{LkH} \alpha 198 \mathrm{~B}$ and they also discovered a suspected bow shock 39" southeast of this source. The bow shock (their knot B), HH 161 and its tail are all aligned and appear to constitute a one-sided outflow from $\mathrm{LkH} \alpha 198 \mathrm{~B}$. To date no counterflow has been seen. CRB also found a number of faint $\mathrm{HH}$ knots (HH 164 C, D and E - see inset in Fig. 1) with the same PA from LkH $\alpha 198$ as HH 461. Thus HH 164 C, D and E and HH 461 appears to delineate an outflow from $\mathrm{LkH} \alpha 198$. To the north they also discovered a faint knot (HH $164 \mathrm{~F})$ which may be a tracer of the counterflow from $\mathrm{LkH} \alpha 198$. Finally to the east of V376 Cas a number of bright HH emission knots, HH 162, were also found by CRB. These knots were seen again in our images, although their origin remains unclear.

Two of the three $\mathrm{HH}$ complexes found here are extensions of the outflow from LkH $\alpha 198$ discovered by CRB. HH 801 has the same PA of $340^{\circ}$ with respect to $\mathrm{LkH} \alpha 198$ as $\mathrm{HH} 164 \mathrm{~F}$ and is therefore almost certainly part of the same flow. It appears to be an asymmetrical bow shock of which we primarily see the western wing (B in Fig. 2) some 45" in length. There are also a 


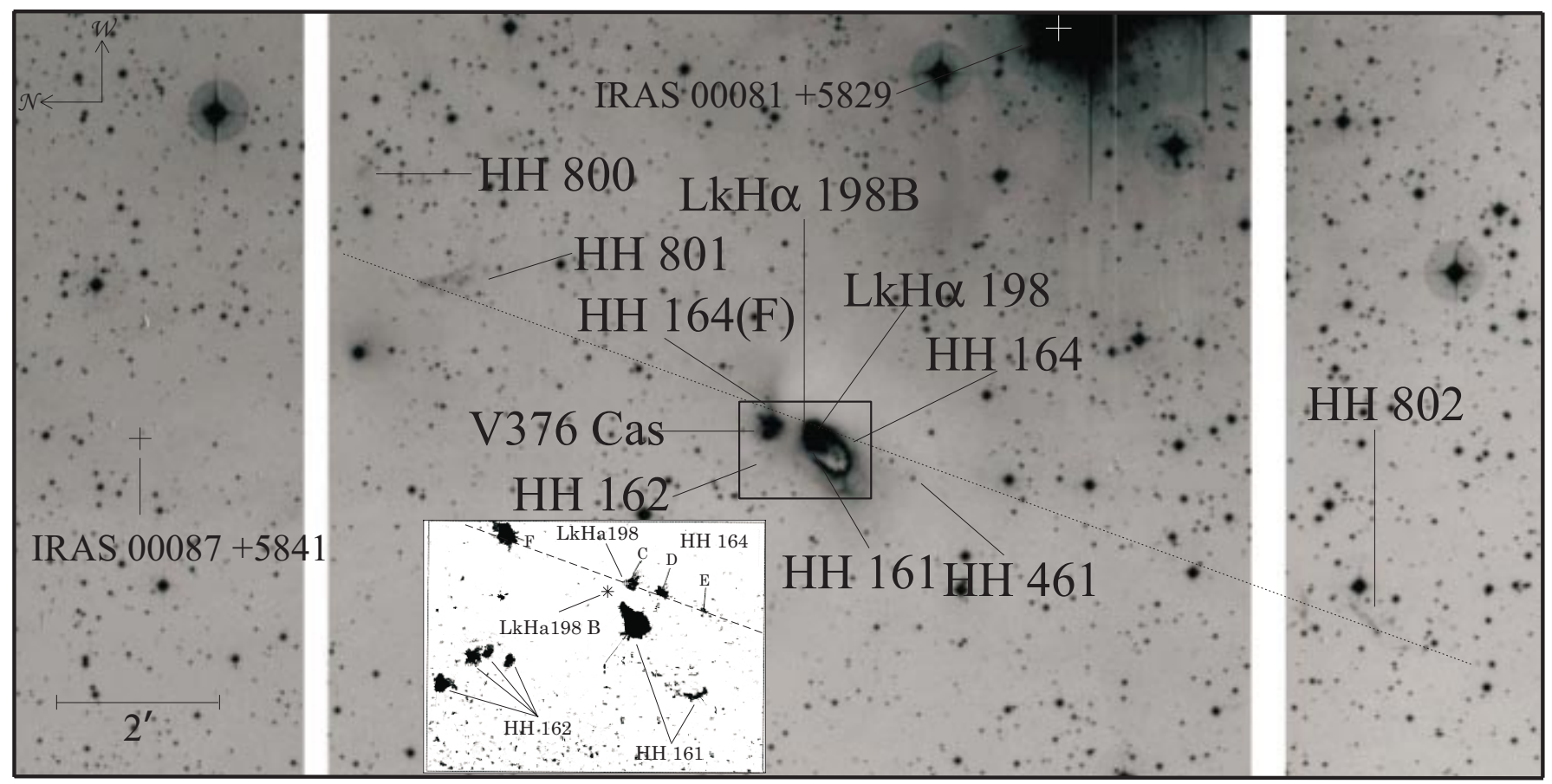

Fig. 1. $L k H \alpha 198$ [SII]: Mosaic of the entire outflow around LkH $\alpha 198$, including the three newly discovered HH complexes with the main outflow axis delineated by a dotted line. For all images relating to $\mathrm{LkH} \alpha 198$ (Figs. 1-3) north is to the left and west to the top but for all subsequent images, north is to the top unless specified otherwise. The continuum subtracted [SII] image of Corcoran et al. (1995) is inset, showing the HH 164 knots (C, D, E and F), most of which are not seen in our [SII] image due to the presence of the reflection nebula. Here, the position of $\mathrm{LkHa} 198$ is indicated by a white cross slightly east of knot C. The PA of HH 164 at $340^{\circ}$ (Corcoran et al. 1995) is marked on the inset and it can be seen from the outflow axis on the main image that the PA of the extended outflow is also at $340^{\circ}$. There are two known optically invisible IRAS sources in the region and their positions are marked. The white strips delineate gaps in the WFC CCD mosaic.

number of more easterly knots, C-F. Knot A may also be part of the western wing.

In the diametrically opposite direction from $\mathrm{HH} 801$ through $\mathrm{LkH} \alpha 198$ we find $\mathrm{HH} 802$. It consists of a number of features, A-I (Fig. 3) which at first sight look somewhat chaotic. Feature E, however, like its counterpart in HH 801, could be the western wing of an asymmetrical bow shock. The total length of HH 802 is some $2^{\prime}$. It has a PA of $160^{\circ}$ with respect to $\mathrm{LkH} \alpha 198$ and it is aligned with knots C, D and $\mathrm{E}(\mathrm{CRB})$ of HH 164 and HH 461 i.e. it is the counterflow of HH 801 and HH 164 Knot F. The furthest knot in HH 802 (Knot I) is at a distance of 8.16 (1.4 pc) from $\mathrm{LkH} \alpha 198$, implying the total projected extent of the flow, from HH 801 to HH 802, is some 2.3 pc.

HH 800 to the northwest of HH 801 is unlikely to be part of the HH 801-HH 802 outflow unless the outflow direction has changed abruptly. Although changes in flow direction have been observed in other parsec-scale outflows (Reipurth \& Bally 2001), they tend to be more gradual. Moreover HH 802 is even further from $\mathrm{LkH} \alpha 198$ than $\mathrm{HH} 800$ (at least in projection) and the flow associated with the former appears to have maintained a constant outflow direction. We should also add that HH 800 is probably not part of the counterflow from $\mathrm{LkH} \alpha 198 \mathrm{~B}$ as its outflow is at a PA of $132^{\circ}$, while $\mathrm{HH} 800$ is at $\sim 330^{\circ}$ with respect to this source. We cannot however exclude the possibility that the outflow axis may have swung through $18^{\circ}$. Finally either of the two optically invisible IRAS sources in the region

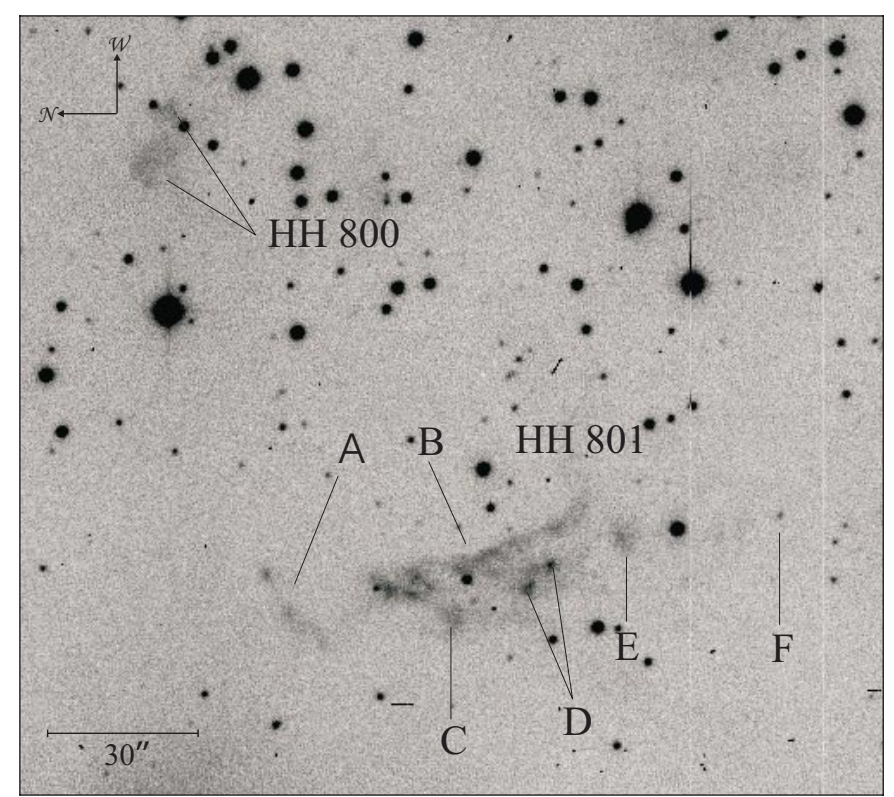

Fig. 2. $L k H \alpha 198$ [SII]: HH 800 and HH 801 showing various features referred to in the text. The knotty morphology of HH 801 is clearly seen here.

(Fig. 1) could be its driving source. IRAS completeness in this region is of the order of $5 L_{\odot}$. Proper motion studies would clearly help to identify its origin. 


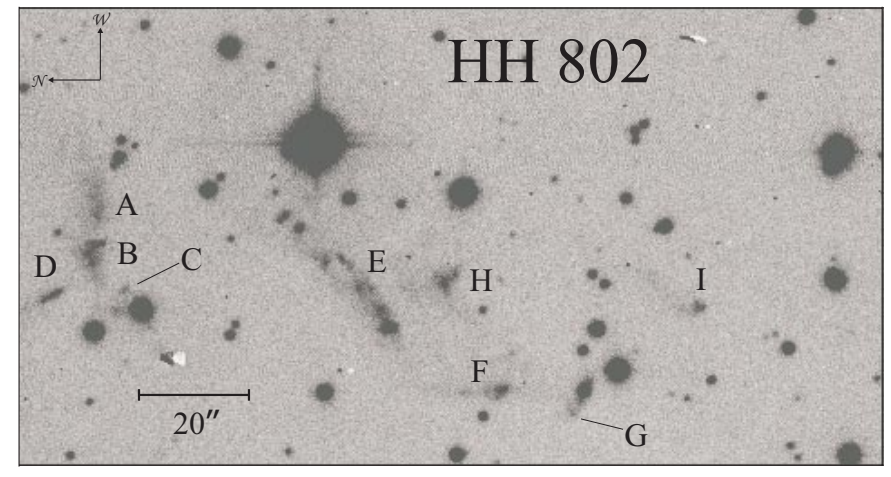

Fig. 3. $L k H \alpha 198$ [SII]: HH 802, to the southeast of $\mathrm{LkH} \alpha 198$.

\section{2. $1548 C 27$}

The cometary-shaped reflection nebula $1548 \mathrm{C} 27$, and its associated $\mathrm{H} \alpha$ emission line jet (HH 165) were first noted by Craine et al. (1981). The optical jet, at a PA of $54^{\circ}$, was later confirmed by Mundt et al. (1984). A low-mass, poorly collimated molecular outflow was also observed in the region by Dent \& Aspin (1992).

Near-infrared photometry in the immediate vicinity of $1548 \mathrm{C} 27$ by Vilchez et al. (1989) yielded two sources. One of these appears to be a foreground star but the other, IRS 1, is located near the apex of the nebula, and they suggest this to be the driving source of HH 165 (see Fig. 4). The IRAS PSC (point source catalogue) shows IRAS $19407+2316$ to be located close to, but not coincident with, IRS 1. Vilchez et al. (1989) however conclude that both near- and far-infrared sources are the same object, which, for convenience, we will refer to here as IRS 1.

The optical jet (HH 165) is very narrow, with a width of $2^{\prime \prime}-3^{\prime \prime}$ and its length is estimated to be $\sim 45^{\prime \prime}$. There is a gap of $\sim 13^{\prime \prime}$ between the source and the jet and two bright knots are visible at 23" and 29" from the source (Mundt et al. 1984). The kinematic distance of 1548 C27 is $2.4 \mathrm{kpc}$ Dent \& Aspin (1992) which implies a luminosity for IRS 1 of approximately $580 L_{\odot}$.

Another star S2, 10" northeast of $1548 \mathrm{C} 27$, was found by Scarrott et al. (1991). This star illuminates the nebula along with IRS 1. Scarrott et al. (1991) suggest HH 165 curves towards this star, implying that it is the driving source, however we find no evidence in our images to support this idea.

HH 365 to the northeast of $1548 \mathrm{C} 27$ was briefly referred to by Alten et al. (1997) as being bow-shaped and possibly associated with $\mathrm{HH} 165$. This object was mentioned in their paper but no image of it was included. Its morphology is clearly seen here in Figs. 4 and 5. HH 365 is 8.13 (5.7 pc) from IRS 1 at a PA of $46^{\circ}$ i.e. close to that of the HH 165 jet. From our images it appears to consist of two bright regions, Knot $\mathrm{A}$ and Feature B that extends to the northwest some $8^{\prime \prime}$ (Fig. 5). The overall shape of Feature B is suggestive of an asymmetrical bow, the axis of which points roughly back towards $1548 \mathrm{C} 27$.

Our survey also revealed number of possible new HH objects (see Fig. 4) although several are very faint. Moreover, it is unclear whether Knots A, B and C (Fig. 6), for example, to the northwest of $\mathrm{HH} 165$ are $\mathrm{HH}$ objects, as there is a lot of contaminating HII nebulosity in the region. However the fact that these lie on the path between HH 165 and HH 365 would

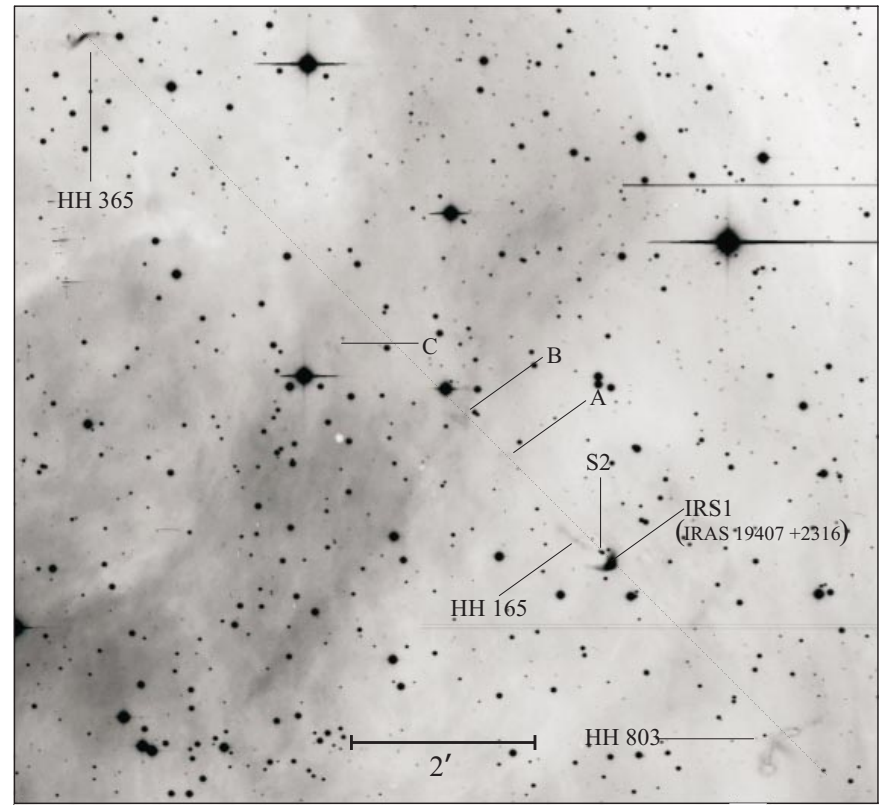

Fig. 4. $1548 C 27 \mathrm{H} \alpha$ : Entire outflow around $1548 \mathrm{C} 27$ IRS 1. Note that north is to the top and east to the left in this and subsequent images. An approximate outflow axis is marked here, at a PA of $\sim 45^{\circ}$, however the outflow appears to be precessing - see text (Sect. 3.2).

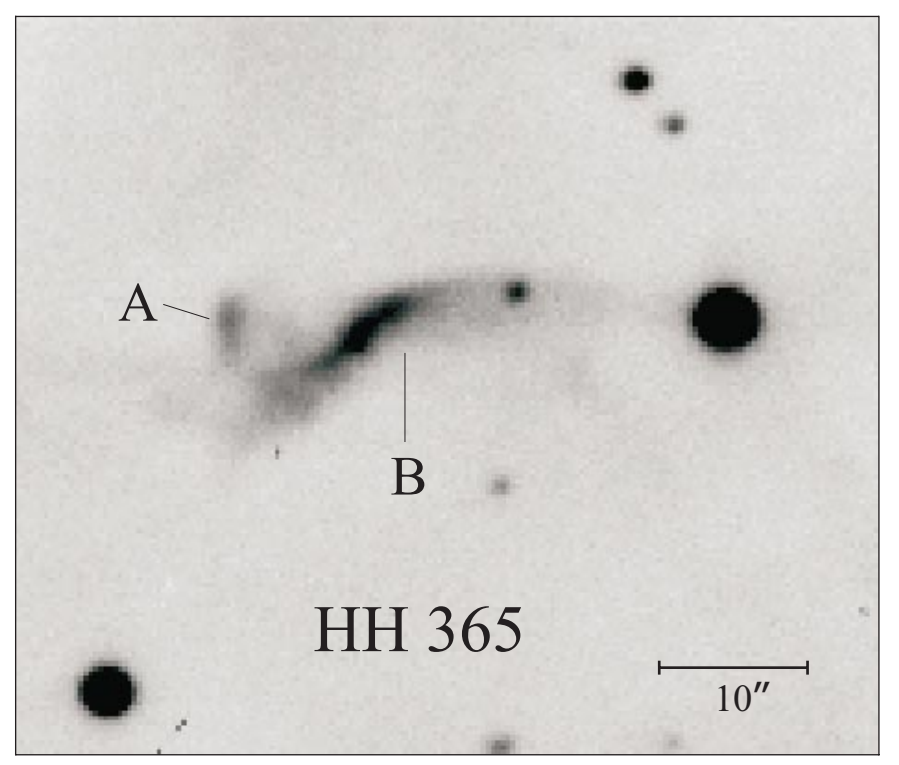

Fig. 5. $1548 C 27 \mathrm{H} \alpha$ : HH 365 , to the northeast of $1548 \mathrm{C} 27$ IRS 1.

suggest they might be. Further study is necessary, however, to determine their nature and for this reason we will desist from assigning them $\mathrm{HH}$ numbers. In any event it seems likely that HH 165 and HH 365 are part of the same outflow from IRS 1 and that Knots A, B and C may also be part of this flow.

A counterjet from IRS 1 was found recently in the nearinfrared (Whelan, private communication). It lies along the same line as $\mathrm{HH} 165$ at a PA of $234^{\circ}$ and extends for at least $5^{\prime \prime}$. A number of faint [FeII] emitting knots were also seen beyond the counterjet. Neither the counterjet nor any of these knots are observed in our optical images, presumably because of extinction. 


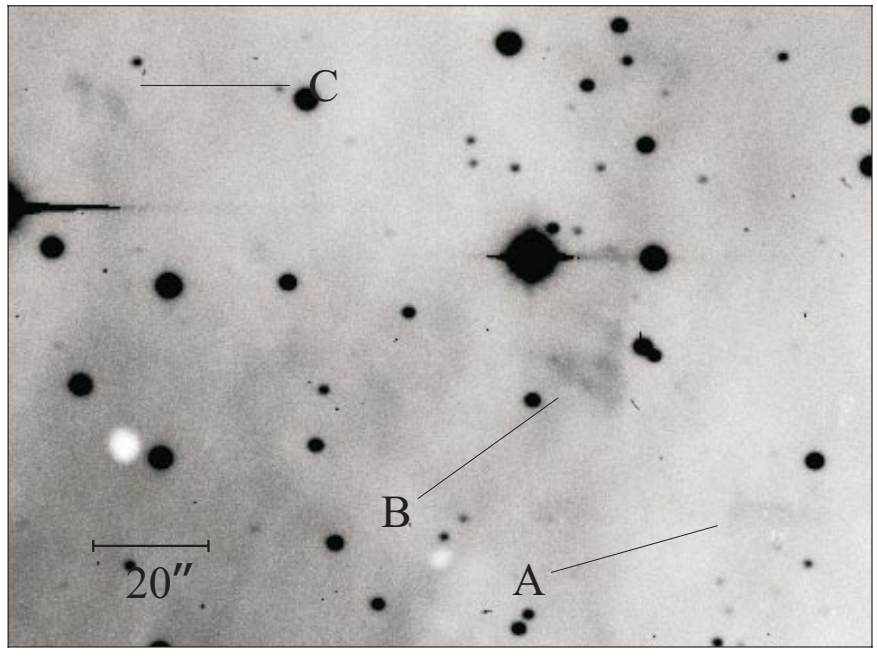

Fig. 6. $1548 C 27 \mathrm{H} \alpha$ : Possible HH knots A, B and C to the northwest of $\mathrm{HH} 165$.

HH 803 (Fig. 7), 2!63 (1.85 pc) southeast of IRS 1 at a PA of $223^{\circ}$, has a very interesting morphology. It appears to be bow-shaped but convex towards IRS 1 . It is $50^{\prime \prime}$ in width and contains a 13" "jet-like" feature bisecting the bow. The "ring" at the southern edge of the bow and the diffuse appearance of Knot A to the north add to the complexity of this object. Note that the jet-like feature does not quite point back towards IRS 1 . We should also add that apart from IRS 1 , no other IRAS sources were found in the region strengthening the possibility that $\mathrm{HH} 803$ is driven by IRS 1 .

If we include Knots $\mathrm{A}, \mathrm{B}$ and $\mathrm{C}$ as part of the HH 165/HH 365/HH 803 outflow then its overall appearance suggests that it may be slowly precessing with shifts in the outflow direction of at least $10^{\circ}$. The sense of precession (i.e. point-like symmetry through IRS 1) to the northeast is also consistent with the position of $\mathrm{HH} 803$ to the southwest.

\subsection{The IRAS $19395+2313$ region}

A number of $\mathrm{HH}$ objects and possible sources were found in this region (see Fig. 8), which lies approximately $18^{\prime}$ west of $1548 \mathrm{C} 27$ and is also in the vicinity of the young open cluster NGC 6823. It is highly unlikely that any of these newlydiscovered HH objects are part of the 1548C27 IRS 1 outflow although we will assume they are at the same distance, i.e. $2.4 \mathrm{kpc}$.

Two of the newly discovered $\mathrm{HH}$ objects are found close together - HH 806 is $30^{\prime \prime}$ west of HH 807 (Fig. 8). The region between them coincides with a gap in the CCD mosaic although a cursory inspection of the Palomar Sky Survey Red (E) plate shows there is a star in the "gap", ALS 10422 or IRAS $19399+2312$ (at $19^{\mathrm{h}} 42^{\mathrm{m}} 05.5^{\mathrm{s}}+23^{\circ} 18^{\prime} 59^{\prime \prime}$, J2000) which, at first sight, might be a possible HH driving source. This object is however an AGB star (Parthasarathy et al. 2000) so we can disregard it. We also see on the Palomar Sky Survey red (E) plate, a conical nebula $236^{\prime \prime}$ southeast of HH 806 (at $19^{\mathrm{h}} 42^{\mathrm{m}} 02.12^{\mathrm{s}}+23^{\circ} 19^{\prime} 30^{\prime \prime}, \mathrm{J} 2000$ ) which may be associated with the driving source of this object. In fact HH 806 lies

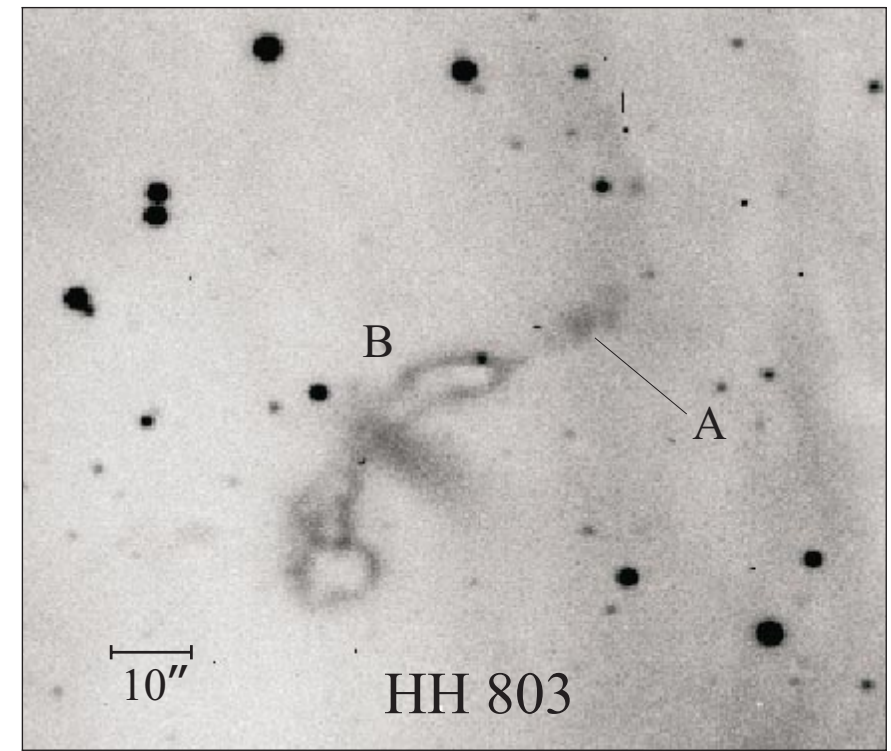

Fig. 7. $1548 C 27 \mathrm{H \alpha}$ : The morphology of $\mathrm{HH} 803$ is clearly seen in optical images.

along the major axis of this conical nebula. Although this nebula is not seen in Fig. 8, its position is marked. There is no obvious driving source for $\mathrm{HH} 807$.

HH 805 has an interesting morphology (Fig. 9) and IRAS $19395+2313$ seems an obvious candidate to be its driving source given its position. We have estimated the luminosity of IRAS $19395+2313$ to be $\sim 320 L_{\odot}$. The angular extent of HH 805 is approximately $45^{\prime \prime}(0.5 \mathrm{pc})$ and it can be seen from Fig. 9 that this outflow is poorly collimated. Morphologically it appears to have a knotty ring-like structure and is reminiscent of the HH complex associated with V380 Ori (Corcoran \& Ray 1995). HH 804 (Fig. 8) may be part of the counterflow from IRAS $19395+2313$ although we emphasise that this association is highly uncertain. It is at a PA of $80^{\circ}$ with respect to the latter. HH 804 is at a distance of 6.3 from IRAS 19395+2313, implying the total projected extent of the flow, assuming HH 804 is part of it, is $\sim 5 \mathrm{pc}$.

\section{4. $L k H \alpha 233$}

$\mathrm{LkH} \alpha 233$ is an A5e-type pre-main sequence star (Corcoran \& Ray 1997) at a distance of $880 \mathrm{pc}$ and is associated with a bipolar nebula that is approximately $0.1 \mathrm{pc}$ in size (Calvet \& Cohen 1978; Staude \& Elsasser 1993). The nebula has a distinct Xlike morphology with bright reflection limbs at $50^{\circ} / 90^{\circ}$ and $230^{\circ} / 270^{\circ}$.

The discovery by Leinert et al. (1993) of a light scattering "halo" $\sim 1$ " in size around $\mathrm{LkH} \alpha 233$ led them to suggest that the star is highly embedded and optically visible largely through scattered light. Corcoran \& Ray (1998) discovered a bipolar jet and counterjet (HH 398) spectroscopically at PAs of approximately $245^{\circ}$ and $65^{\circ}$ that bisect the $\mathrm{X}$-shaped nebula. In their spectrograms, the redshifted counterjet is seen to begin $0^{\prime \prime} .7$ from the centre of the stellar continuum, whereas the blueshifted jet can be traced right back to the continuum peak. The jet, and counterjet, can be seen in our continuum subtracted 


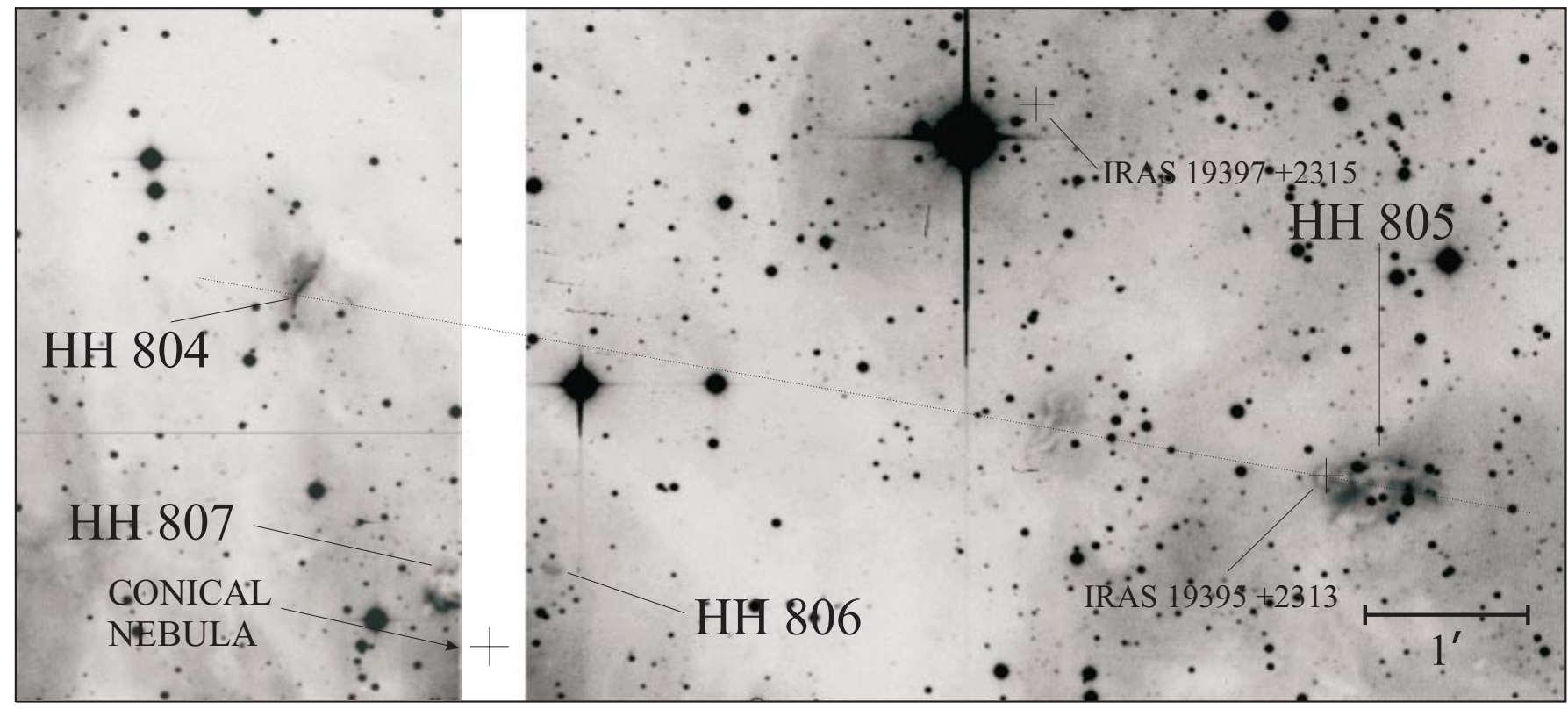

Fig. 8. IRAS 19395+2313 H $\alpha$ : The region around IRAS $19395+2313$, including all known additional IRAS sources in its vicinity. Note that IRAS completeness at this distance is $\geq 100 L_{\odot}$. Four newly discovered HH objects are marked. The position of the conical nebula, visible on the Palomar Sky Survey Red (E) plate and which might be associated with the driving source for HH 806, is indicated. The possible outflow from IRAS $19395+2313$ is marked with a dotted line at a PA of $80^{\circ}$.

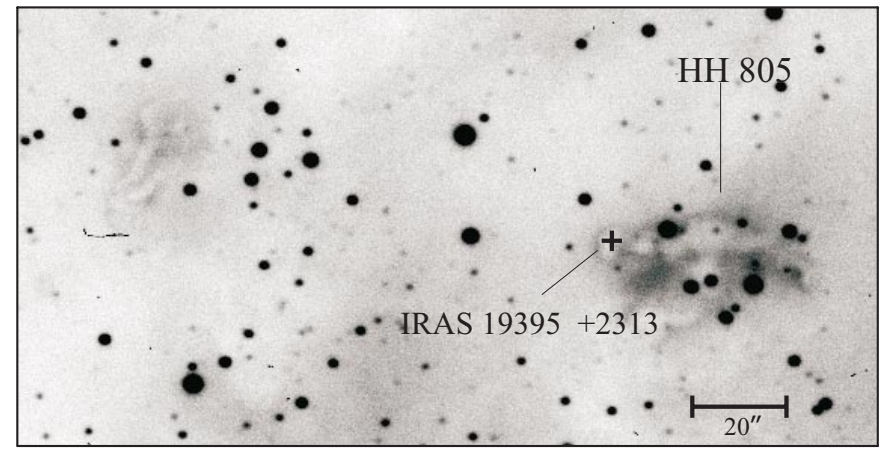

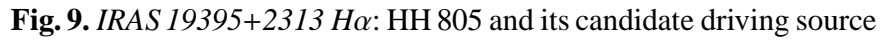
IRAS $19395+2313$.

image (Fig. 12) along with a number of $\mathrm{HH}$ objects along the outflow direction.

Evidence for the presence of a large "polarisation disk" centred on $\mathrm{LkH} \alpha 233$ with a radius of about $15000 \mathrm{AU}$ was found by Aspin et al. (1985). The position angle of this disk is about $155^{\circ}$, which places it perpendicular to the observed optical outflow. The existence of this "polarisation disk" combined with the fact that the counterjet is not seen spectroscopically close to the star leads to the conclusion that there is a circumstellar disk present that obscures the beginning of the receding flow.

Close to $\mathrm{LkH} \alpha 233$ the [SII] emission can be resolved into two velocity components (Corcoran \& Ray 1998). The high velocity component is identified with the optical jet while the low velocity component, which extends to less than $2^{\prime \prime}$ from the star, may be modelled as a rotationally broadened disk wind (see Kwan \& Tademaru 1995).
This survey revealed a number of previously unknown $\mathrm{HH}$ objects in the vicinity of $\mathrm{LkH} \alpha 233$. It is possible that not all of these objects can be attributed to $\mathrm{LkH} \alpha 233$ and the positions of a nearby IRAS source, IRAS $22317+4024$, complicates our analysis (see Fig. 10). Candidate driving sources for all the new $\mathrm{HH}$ objects are suggested here.

Continuum subtracted images of the nebula surrounding $\mathrm{LkH} \alpha 233$ reveal a number of $\mathrm{HH}$ features which are not seen, at least so clearly, in the [SII] image alone. Figure 12 shows the first optical images of an $\sim 7^{\prime \prime}$ jet emerging from the $\mathrm{LkH} \alpha 233$ nebula at a PA of $\sim 248^{\circ}$, which is relatively close to the PA of the jet as spectroscopically determined by Corcoran \& Ray (1998). A possible counterjet to the northeast of $\mathrm{LkH} \alpha 233$ is also seen in this image. But it is difficult to determine whether this is actually a counterjet or simply a residual of the continuum subtraction process. There are two other emission knots to the southwest of the source. The first of these, HH 808, is situated close to a diffraction spike from a bright star to the west of $\mathrm{LkH} \alpha 233$. HH 808 is 1'.05 from the source at a PA of $250^{\circ}$. The second knot, $\mathrm{HH} 809$, is 2.2 from the source at a PA of $241^{\circ}$. Two other objects were seen to the northeast of $\mathrm{LkH} \alpha 233$ at a distance of $\sim 2^{\prime}$, the first has a PA of $63^{\circ}$ and the second is at $65^{\circ}$. These objects are outside the area shown in Fig. 12 and it is unclear at present whether these are HH objects.

Also to the northeast of $\mathrm{LkH} \alpha 233$, we discovered $\mathrm{HH} 814$ (Fig. 10) at a distance of 5'.18 (1.3 pc). The morphology of this object may be studied more clearly in the continuum subtracted image inset in Fig. 10. HH 814 appears to be a broad bow shock facing back towards $\mathrm{LkH} \alpha 233$ at a PA of $47^{\circ}$ with respect to $\mathrm{LkH} \alpha 233$. Corcoran \& Ray (1998) determined a PA of approximately $65^{\circ}$ for the counterjet, suggesting that if $\mathrm{HH} 814$ is part of the same flow, its direction has changed by $\sim 20^{\circ}$. 


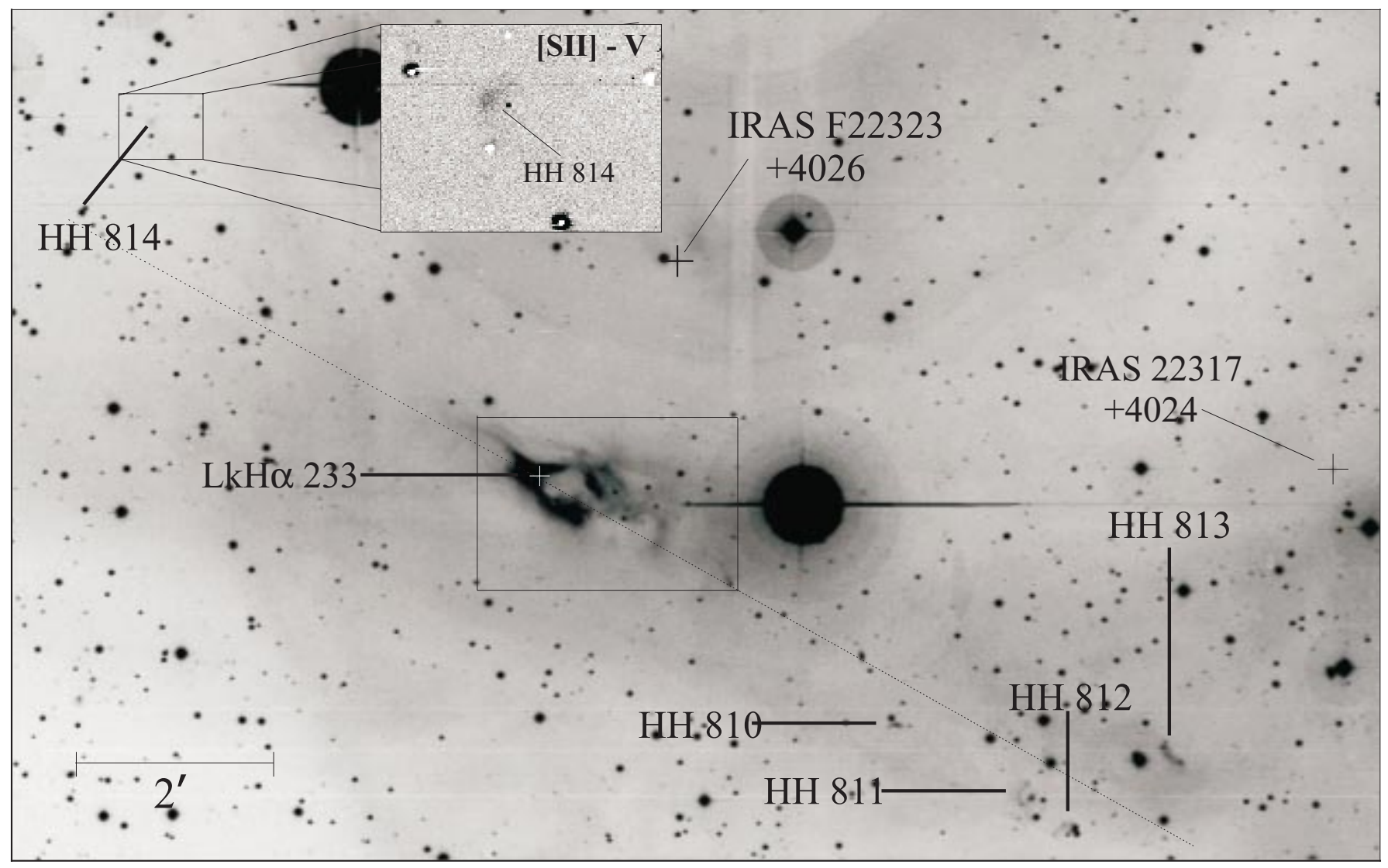

Fig. 10. $L k H \alpha 233$ [SII]: The entire outflow around $\mathrm{LkH} \alpha 233$ including all known optically invisible IRAS sources in the region. The major axis of the outflow at $62^{\circ}$ through the source is indicated by a straight line. The continuum subtracted ([SII]-V) image in the top right corner shows HH 814 more clearly. The area around $\mathrm{LkH} \alpha 233$ is marked by a box and is seen in more detail in Fig. 12.

Note however that the PA determined by Corcoran \& Ray (1998) is very crude as it was deduced by slit sampling at various PA's.

A number of other objects were discovered to the southwest of LkH $\alpha 233$ (see Fig. 11). HH 810, HH 811 and HH 812, are at $4.5(1.2 \mathrm{pc}), 6^{\prime}(1.5 \mathrm{pc})$ and $6.5(1.7 \mathrm{pc})$ respectively from $\mathrm{LkH} \alpha 233$, all at a $\mathrm{PA}$ of $236^{\circ}$. $\mathrm{HH} 813$, at a distance of $7^{\prime}(1.8 \mathrm{pc})$ has a $\mathrm{PA}$ of $247^{\circ}$ with respect to $\mathrm{LkH} \alpha 233$. Considering the possibility that outflows from higher mass stars may not be as collimated as those from low mass stars, HH 810-HH 814 could be the optical tracers of the edges of a moderately collimated flow driven by $\mathrm{LkH} \alpha 233$. The axis of this outflow with respect to $\mathrm{LkH} \alpha 233$ is $\sim 62^{\circ}$ (marked on Fig. 10), which agrees well with the estimate of the PA of the jet closer to the source. It is also possible that HH 813 is a bow shock facing back towards IRAS 22317+4024 (Fig. 11). Proper motion studies could conclusively determine whether this is the case.

\subsection{The NGC 7129 region}

A large number of YSOs are known in the NGC 7129 cluster which lies at a distance of $1.25 \mathrm{kpc}$ (Shevchenko \& Yakubov 1989). Aside from optically visible young stars such as $\mathrm{LkH} \alpha 234$, there are many embedded ones. For example, a $160 \mu \mathrm{m}$ survey by Bechis et al. (1978) revealed two far infrared sources and although one of them is spatially coincident with $\mathrm{LkH} \alpha 234$ (FIRS 1), the other is $3^{\prime}$ further south (FIRS 2) and optically invisible. Additional infrared (Weintraub et al. 1994; Cabrit et al. 1997) and sub-millimetre sources (Fuente et al. 2001) are also known.

Associated with these sources one finds the usual host of phenomena typical of star formation including reflection nebulae (Bertout 1987), molecular outflows (Edwards \& Snell 1983; Bertout 1987; Mitchell \& Matthews 1994), HH objects (Ray 1987; Miranda et al. 1993), HH jets (Ray 1987; Ray et al. 1990; Cabrit et al. 1997) and shocked $\mathrm{H}_{2}$ flows (Eislöffel 2000).

Looking at the distribution of previously known $\mathrm{HH}$ objects in the region, and the new ones discovered here (see below), one gets the impression (see Figs. 13 and 14) that the primary outflow axis, or axes, is roughly in a northeast to southwest direction and centred on the cluster core. Caution however is necessary. Proper motions studies have shown that some HH objects like GGD 32 and HH 103 are not moving to the southwest, as one might suspect, but instead to the west (Ray et al. 1990). Moreover others, like GGD 34 (Gómez de Castro \& Robles 1999) and possibly GGD 33 (Cohen \& Schwartz 1983; Goodrich 1986), have their own sources outside the cluster core.

The HH 815 complex (Fig. 15) is over $1^{\prime}$ in size and is at a distance of $11^{\prime}$ (4 pc) from $\mathrm{LkH} \alpha 234$. The three emission regions in $\mathrm{HH} 815$ (A, B and $\mathrm{C}$ ) appear to form the edges of a large bow shock that is concave towards the cluster core. HH 816 (Fig. 16) may be another bow trailed by a series of 


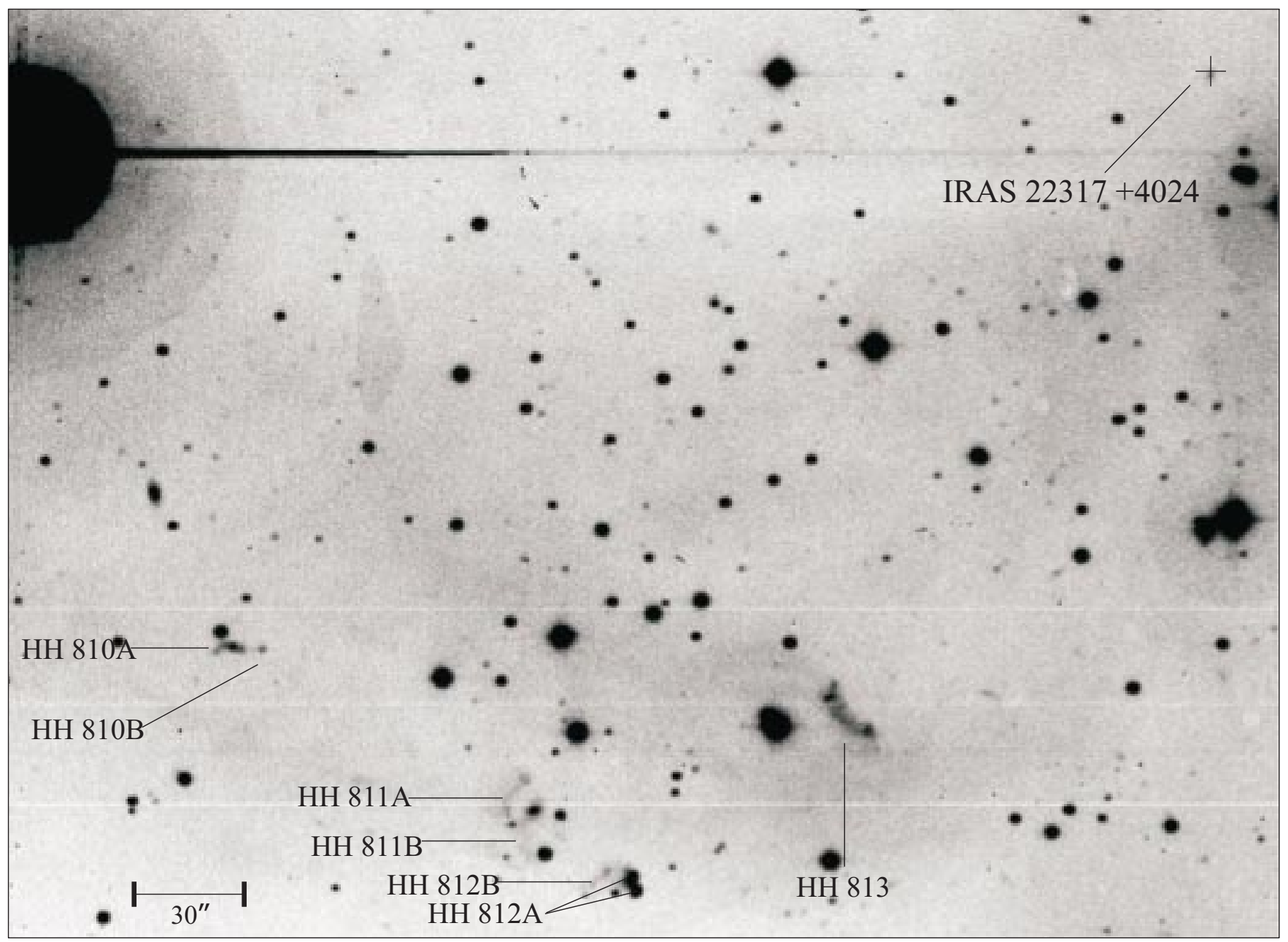

Fig. 11. $L k H \alpha 233$ [SII]: HH 810-HH 813, to the southwest of $\mathrm{LkH} \alpha 233$.

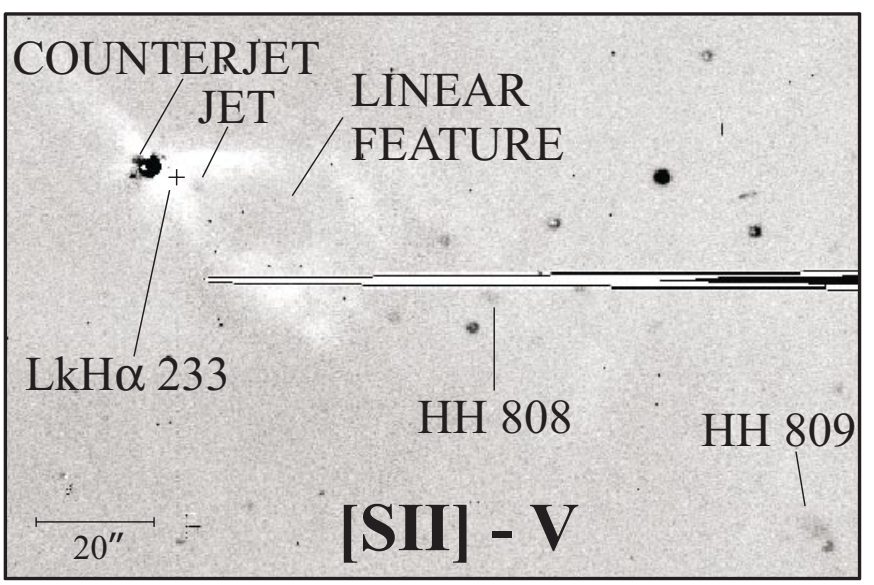

Fig. 12. $L k H \alpha 233$ [SII]: Continuum subtracted image of the centre of the $\mathrm{LkH} \alpha 233$ nebula. The jet is optically visible here, along with a number of other emission features close to the source including HH 808 and HH 809.

faint HH knots i.e. HH 817-HH 820. HH 821, 15" north of GGD 35, is aligned with HH 819 and HH 816 and so could form part of the same flow. We note also that HH 105, is on the same axis from the cluster core and that no source for $\mathrm{HH} 105$, at least in its immediate vicinity, is known.

The most distant $\mathrm{HH}$ object to the southwest of the cluster discovered by us is HH 822 (Fig. 17). It is at a PA of $238^{\circ}$ with respect to $\mathrm{LkH} \alpha 234$ and the morphology of its brightest component (HH 822 A), i.e. a bow concave towards the cluster, suggests it is part of an outflow that originated there. An additional knot close to $\mathrm{HH} 103$ was also found and we shall refer to it as HH $103 \mathrm{~A}$ (Fig. 13). Given its location, it seems likely it is part of the same flow that drives HH 822. Moreover faint emission can be seen linking $\mathrm{HH} 103 \mathrm{~A}$ to $\mathrm{HH} 822 \mathrm{~A}$ reinforcing this conclusion. Finally we add that $\mathrm{HH} 822 \mathrm{~B}$ is at a distance of 10'7 (3.9 pc) from $\mathrm{LkH} \alpha 234$.

The large number of sources in this region makes it extremely difficult, without detailed kinematic studies, to determine the origin of individual $\mathrm{HH}$ complexes. As previously mentioned there are a number of low mass YSOs, like the one that drives GGD 34, present and this complicates our analysis. That said, it seems likely, purely on morphological grounds as well as location, that many of the newly discovered $\mathrm{HH}$ objects are driven by a source(s) in the cluster core. Because of their large distance from the core, however, it may prove impossible, even with good proper motion data, to determine their precise origin. Several possible candidates exist including $\mathrm{LkH} \alpha 234$, 


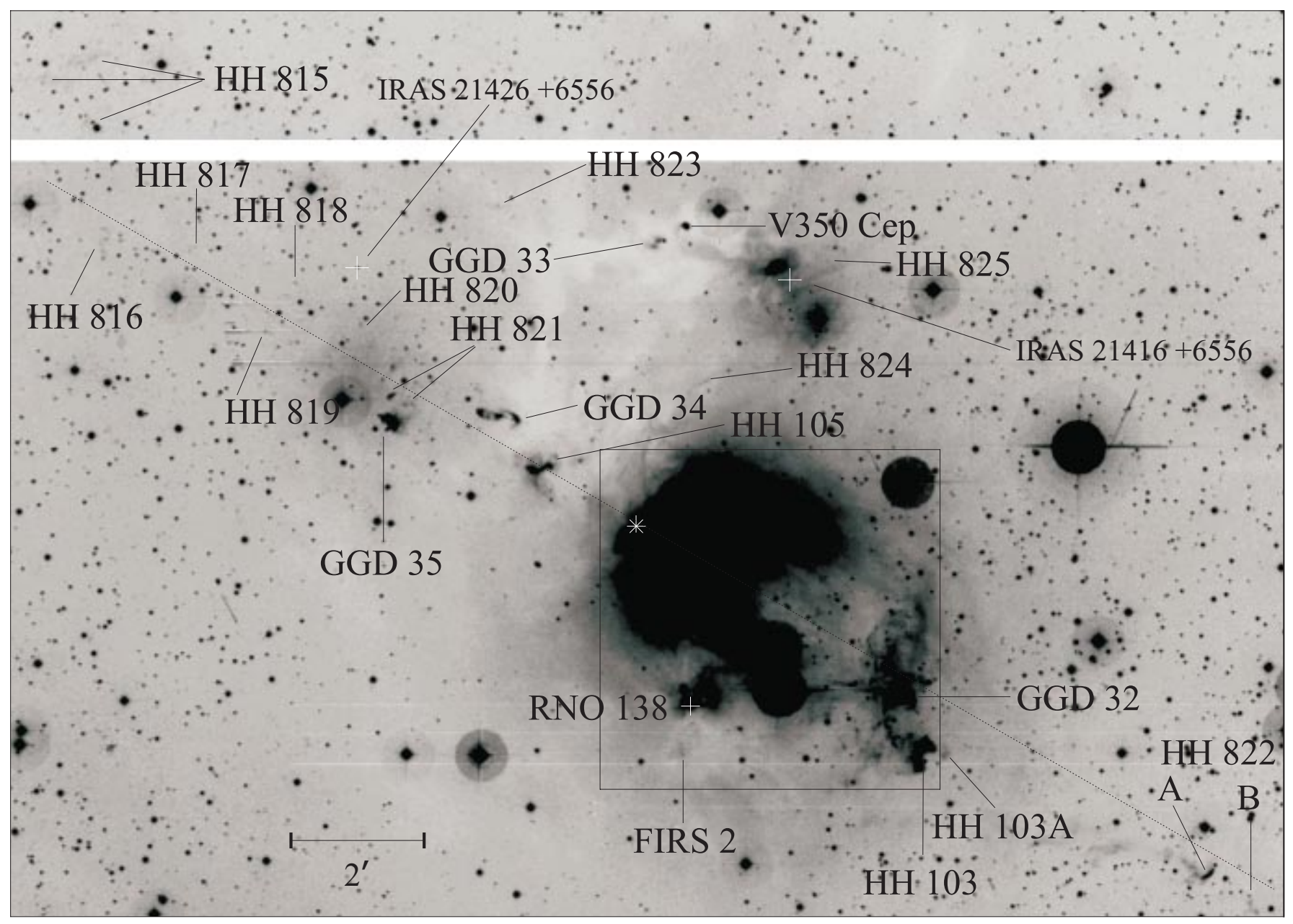

Fig. 13. $L k H \alpha 234$ [SII]: Mosaic of the entire outflow around $\mathrm{LkH} \alpha 234$. The position of $\mathrm{LkH} \alpha 234$ is marked with a white star - the area surrounding $\mathrm{LkH} \alpha 234$ indicated by the box can be seen more clearly in Fig. 14. The dotted line marks the primary outflow axis flow at $60^{\circ} / 240^{\circ}$. There is an optically invisible IRAS source in the cluster, IRAS $21418+6552$, the position of which is marked with a white cross in Fig. 14. Other known infrared sources in the region are also indicated.

IRS 6 and FIRS 1-MM1. If we draw an imaginary axis through the core at a PA of $60^{\circ} / 240^{\circ}$ it roughly delineates the region where most of the new $\mathrm{HH}$ objects, $\mathrm{HH} 816$ to $\mathrm{HH} 822$, are located. Assuming we are dealing with one outflow here, that originates in the core, then its overall angular size is 21.8 i.e. $\sim 8 \mathrm{pc}$ in projected length.

There are three other $\mathrm{HH}$ objects which are not situated along the major axis marked in Fig. 13. HH 824 and HH 825 are located on either side of IRAS $21416+6556$ at $143^{\circ} / 323^{\circ}$ with respect to this source, suggesting a possible bipolar outflow driven by IRAS $21416+6556$. These objects are $2^{\prime}$ and $51^{\prime \prime}$ respectively from IRAS $21416+6556$. The driving source of HH 823 is unclear as is its association with any of the other outflows in this region.

\section{Discussion}

\subsection{Overall lengths}

It has been stated, albeit somewhat tongue-in-cheek, that the apparent length of optical outflows from YSOs used to be a function of CCD chip size! Early chips were small and sampled only a small angular patch of the sky. This, in combination with the episodic nature of the flows themselves, conspired to suggest outflow lengths measured in tens of thousands of AU rather than parsecs. In a number of cases however CCD mosaicing (Ray 1987) did hint that some flows were at least in the parsec league. Large format CCDs in focal-plane mosaics can cover fields of view larger than $30^{\prime}$ which, at a distance of $1 \mathrm{kpc}$, corresponds to more than $8.7 \mathrm{pc}$. Flows can therefore be detected to beyond the peripheries of their parent cloud.

Table 2 lists parameters such as distance, source luminosity, outflow length, cloud size and degree of collimation for both the intermediate-mass sources discussed here and several wellstudied outflows powered by both intermediate- and low-mass YSOs. In some regions, the projected lengths of the outflows are similar to the sizes of the clouds from which they emerge. This correlation of length scales is to be expected and there should also be a tendency for shocks to be seen near the cloud edges where extinction by dust is minimal.

If we assume an average tangential velocity for outflows of $100 \mathrm{~km} \mathrm{~s}^{-1}$, in $10^{5} \mathrm{yrs}$ material originally at the source will be transported $\sim 10$ pc i.e. typically to the edge of the clouds we 
Table 2. Parameters of newly discovered, and some previously known, parsec-scale outflows from low- and intermediate-mass YSOs.

\begin{tabular}{llllllllll}
\hline \hline Source & $\begin{array}{l}\text { Distance } \\
(\mathrm{pc})\end{array}$ & Ref. & $\begin{array}{l}L_{\text {bol }} \\
/ L_{\odot}\end{array}$ & Ref. & $\begin{array}{l}\text { Outflow } \\
\text { length }(\mathrm{pc})\end{array}$ & Ref. & $\begin{array}{l}\text { Associated } \\
\text { Cloud }\end{array}$ & $\begin{array}{l}\text { Cloud size }^{a} \\
(\mathrm{pc})\end{array}$ & $\begin{array}{l}\theta_{\text {flow }}{ }^{b} \\
\left({ }^{\circ}\right)\end{array}$ \\
\hline LkH $\alpha$ 198 & 600 & 2 & $\geq 160$ & 2 & 2.3 & 1 & L1265 & 2 & 2.8 \\
1548C27 IRS 1 & 2400 & 3 & 580 & 3 & 7.5 & 1 & NGC 6823 & 33 & 11.6 \\
IRAS 19395+2313 & 2400 & 3 & 320 & 1 & 5 & 1 & NGC 6823 & 33 & 30.2 \\
LkH $\alpha$ 233 & 880 & 4 & $\geq 121$ & 4 & 3.1 & 1 & ANON & 3.2 & 6.3 \\
LkH $\alpha$ 234 & 1250 & 5 & 1200 & 6 & 8 & 1 & NGC 7129 & 5.1 & 6.2 \\
IRAS 18162-2048c & 1700 & 7 & 1700 & 8 & 5.3 & 8 & L291 & 15 & 1 \\
HH 354 IRS & 750 & 9 & 120 & 9 & 2.4 & 10 & L1165 & 1.8 & 4.04 \\
PV Cephei & 500 & 11 & 100 & 12 & 2.6 & 10,13 & L1617 & 28 & 6.7 \\
IRAS 05491+0247 ${ }^{d, e}$ & 460 & 10 & 25 & 10 & 7.7 & 10 & L1617 & 28 & 7.4 \\
HH 1/2 VLA 1 ${ }^{e}$ & 460 & 14 & 50 & 15 & 5.9 & 16 & L1641 & 25 & 10.3 \\
HH 34 IRS & 460 & 14 & 28 & 17 & 3 & 18 & L1641 & 25 \\
\hline
\end{tabular}

${ }^{a}$ This is the diameter of the cloud where diameter $=[$ major axis + minor axis $] / 2$.

${ }^{b} \theta_{\text {flow }}$ is calculated by taking the width of the most distant shock in both the outflow and counterflow and dividing by the projected distance from the source. The mean value of $\theta_{\text {flow }}$ for the outflow and counterflow is given here.

${ }^{c}$ The source of $\mathrm{HH} 80 / \mathrm{HH} 81$.

${ }^{d}$ The source of HH 111 .

${ }^{e}$ These YSOs are low-mass sources and are included here for comparison purposes only.

References : 1. this paper; 2. Chavarria-K. (1985); 3. Dent \& Aspin (1992); 4. Calvet \& Cohen (1978); 5. Shevchenko \& Yakubov (1989); 6. Harvey et al. (1984); 7. Rodriguez et al. (1980); 8. Marti et al. (1993); 9. Schwartz et al. (1991); 10. Reipurth et al. (1997); 11. Cohen et al. (1981); 12. Mundt \& Ray (1994); 13. Gomez et al. (1997); 14. Hester et al. (1998); 15. Harvey et al. (1986); 16 Ogura (1995); 17. Reipurth et al. (1993); 18. Bally \& Devine (1994).

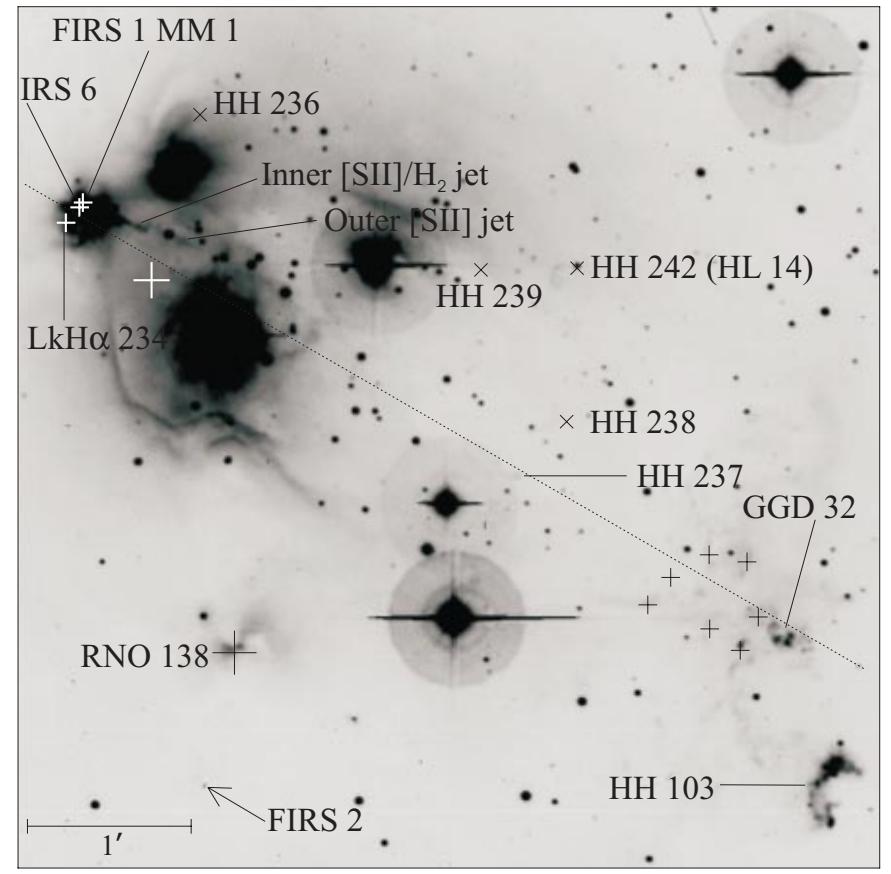

Fig. 14. $L k H \alpha 234$ [SII]: The cluster region (indicated in Fig. 13) including the "inner" and "outer" optical jets (Ray et al. 1990; Cabrit et al. 1997). The contrast has been changed here with respect to Fig. 13 so that more objects within the cluster core are visible. The white cross to the southwest of $\mathrm{LkH} \alpha 234$ marks the position of IRAS 21814+6552. Black crosses and X's are used to mark the positions of HH objects found by Eiroa et al. (1992) and Miranda et al. (1993) respectively. The apparent primary outflow axis through the cluster at $60^{\circ} / 240^{\circ}$ is marked. are studying. Using the evolutionary tracks of Palla \& Stahler (1993), we see that such a period corresponds to about $10 \%$ of the time an average Herbig $\mathrm{Ae} / \mathrm{Be}$ star spends in the premain sequence phase. It follows that only in the case of the more massive YSOs, i.e. those with the shortest evolutionary timescales, do HH outflows represent a "fossil record" of activity over the totality of the outflow phase.

\subsection{Morphology}

HH outflows are episodic: they are clearly not continuous phenomena. For the most part, the shocks we see are generated by supersonic jet material ramming into previously ejected slower gas. This process produces a series of "working surfaces", radiative shock systems that fade with time and therefore with distance from their source. Only the strongest shocks survive to produce dramatic, often chaotic, structures on parsec scales. It has even been suggested that the FU Orionis phenomena may signal the dramatic change in output needed at the source to produce such a shock (Reipurth 1989). Thus the spacing between $\mathrm{HH}$ objects increases with distance from the source at least amongst low mass YSOs (Reipurth \& Bally 2001). Such a trend is also visible here amongst the flows from intermediate mass YSOs such as $\mathrm{LkH} \alpha 198$ and $1548 \mathrm{C} 27$. Another phenomenon that occurs with HH outflows from low mass YSOs is that the shock structures become larger and apparently more chaotic with distance. Again this is something which is replicated in their intermediate mass YSO counterparts.

A phenomenon that is found in parsec-scale outflows from lower mass YSOs is that the flow often exhibits "S" or "C" shape symmetry (Reipurth 1989) possibly as a result of jet 


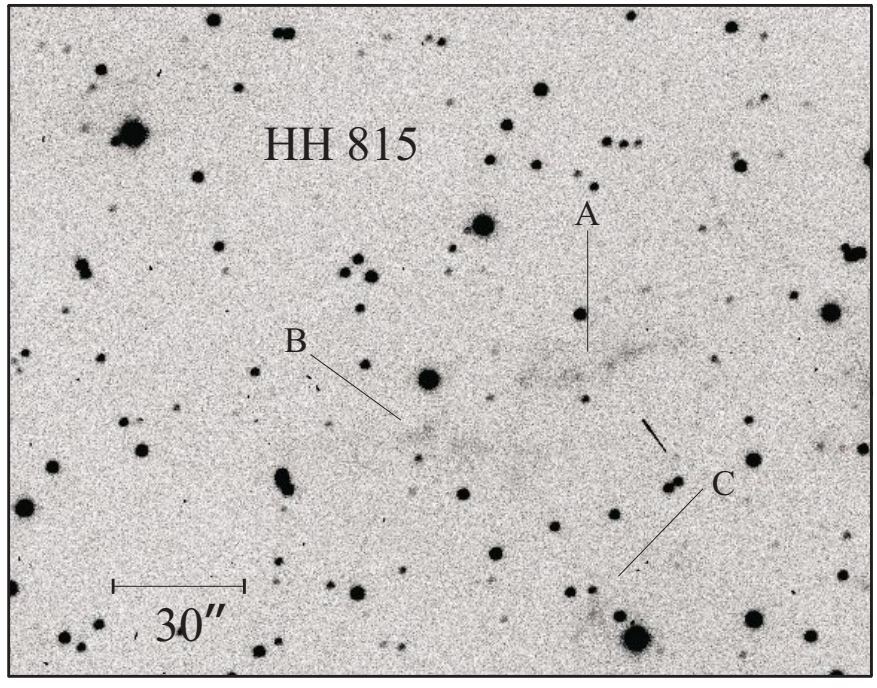

Fig. 15. $L k H \alpha 234$ [SII]: HH 815, to the northeast of the cluster, is the most distant, known, outlying $\mathrm{HH}$ object in this region. Its relative position is seen in Fig. 13.

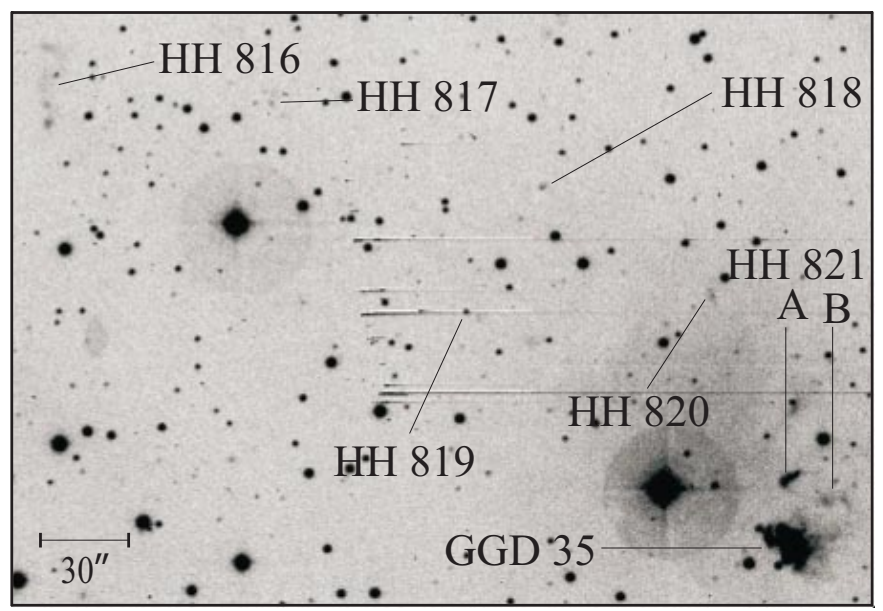

Fig. 16. $L k H \alpha 234$ [SII]: HH 816-HH 821 (A and B), to the northeast of $\mathrm{LkH} \alpha 234$.

precession or source motion through the parent cloud respectively. In our small sample, we do not find any clear-cut examples of either although, as previously mentioned, the parsecscale outflow from 1548C27 IRS 1 may be "S-shaped".

\subsection{Collimation}

As alluded to in the Introduction, one of the most striking differences between parsec-scale outflows from low and massive YSOs is the relative lack of collimation seen in the latter (Shepherd et al. 1997; Hunter et al. 1997; Shepherd et al. 1998). This point is well illustrated by the archetypal example of an outflow from a high luminosity YSO: the Orion IRC2 flow (Allen \& Burton 1993). Its opening angle is $\sim 90^{\circ}$ (Burton $\&$ Allen 1994) which is a sharp contrast to the angles (typically a few degress) seen in outflows from low-mass YSOs.

Table 2 list "final" opening angles for our small sample. Values are determined by dividing the width of the most distant $\mathrm{HH}$ object by its projected source separation. Note

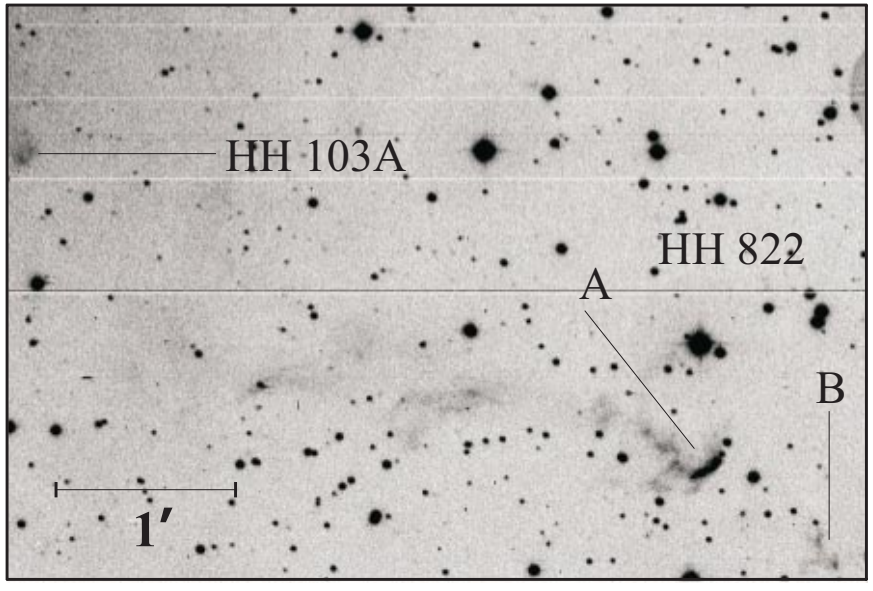

Fig. 17. $\mathrm{LkH \alpha} 234$ [SII]: HH $103 \mathrm{~A}$ and HH 822 to the southwest of $\mathrm{LkH} \alpha 234$.

that as we are using projected separations the quoted values must be upper limits, however our observations only reveal the brightest portions of the shocks and fainter outer parts may be missed, so the opening angles may actually be greater than what is optically observed. Observed opening angles range from $0.9^{\circ}$ to $12^{\circ}$ suggesting a degree of collimation comparable to that seen in outflows from low-mass YSOs. This also suggests that the transition from well-collimated outflows to poorly-collimated outflows occurs at higher masses than the sources observed here.

\subsection{The frequency of blow-outs}

The true size of an outflow, in comparison to that of its parent cloud, is an important factor in determining whether the outflow's energy and momentum is transported into the ISM or remains within the cloud itself. A cursory examination of our data shows a clear tendency for $\mathrm{HH}$ objects to lie close to the edges of the parent cloud or at least close to the edges of clumps. As already mentioned, such an effect is to be expected considering the low extinction near cloud peripheries and the very low ISM densities beyond the cloud boundaries.

More importantly, it is clear that actual outflow timescales are very long in comparison to apparent dynamical ones. Here dynamical timescales are derived by dividing the projected length of an outflow by its estimated tangential velocity. This, and the observation of $\mathrm{HH}$ objects near cloud boundaries, immediately suggests that most, if not all, of the flows studied here have blown-out of their parent cloud.

\subsection{Are outflows a source of cloud turbulence?}

There is plenty of evidence to suggest molecular clouds are turbulent (Ward-Thompson 2002 and references therein) and that the pressure generated by this turbulence is sufficient to prevent clouds from collapsing under their own gravity. It has been shown however that cloud turbulence, even in the presence of magnetic fields, decays too quickly compared to typical cloud lifetimes (Stone et al. 1998) and must therefore 
be somehow replenished. Could outflows be the primary source of turbulence in molecular clouds?

The parsec-scale HH outflows imaged here appear largely well-collimated and therefore one might think they could affect only a narrow cone of ambient material. One has to remember however that these flows are supersonic and that we are viewing only the most highly collimated outflow component. The same flows "imaged" in the CO $J=1 \rightarrow 0$ line would normally appear much less collimated, especially at low velocities. That these flows affect cloud structure on parsec scales is evident from features such as the large CO "cavity" in NGC 7129. No doubt they also affect cloud dynamics. Arce \& Goodman (2001) for example has found that molecular outflows associated with parsec-scale $\mathrm{HH}$ flows can possess kinetic energies comparable to the turbulent and gravitational binding energies of their parent clouds. This proves however only that they are a potentially important source of turbulence. Unfortunately we do not understand the coupling between outflows and their ambient medium well enough to be sure. Numerical simulations (Downes \& Cabrit 2003) are helping to address this problem but further studies are required.

\section{Conclusions}

We have investigated the occurrence of parsec-scale outflows from intermediate-mass YSOs. As is the case with lower mass YSOs, such flows appear to be common and we report the discovery of four here with well defined sources. These include LkH $\alpha 198,1548 C 27$ IRS 1, LkH $\alpha 233$ and IRAS 19395+2313. All of these YSOs, with the exception of the last, were previously known to have small-scale outflow activity.

The region surrounding the Herbig Ae star LkH $\alpha 234$ is cluttered with outflows and candidate sources. Twelve new $\mathrm{HH}$ objects are reported on here, with many of them lying along a preferential axis centred near $\mathrm{LkH} \alpha 234$ and orientated in a northeast-southwest direction. Their morphology suggests that at least some are part of a large-scale flow centred on the core of the NGC 7129 cluster.

Parsec-scale outflows from intermediate-mass YSOs show a number of similarities to those from their low-mass counterparts. In particular:

- their lengths and degree of collimation appear comparable, - they share the same morphological trends such as decreasing frequency, increasing dimension and increasing complexity of $\mathrm{HH}$ emission with distance from the source.

The lengths of these large-scale outflows are usually comparable to the clump size of their associated clouds. As their expected lifetimes are much larger than the apparent dynamical timescales, this suggests that many have "blown-out" of the cloud complex.

Finally, it is evident that the transition from highly collimated jet-like flows to poorly collimated wide-angle outflows such as OMC 1 must lie at higher masses and luminosities than the sources studied here.

Acknowledgements. FMcG and TPR acknowledge support from Enterprise Ireland. JBs research was supported by NSF grant AST-9819820, NASA grant ANG5-8108 (LTSA), and NASA grant NCC2-1052 (CU Center for Astrobiology).

\section{References}

Allen, D. A., \& Burton, M. G. 1993, Nature, 363, 54

Alten, V. P., Bally, J., Devine, D., \& Miller, G. J. 1997, Herbig-Haro Flows and the Birth of Stars, IAU Symp., 182, 51P

Arce, H. G., \& Goodman, A. A. 2001, ApJ, 554, 132

Aspin, C., McCaughrean, M. J., \& McLean, I. S. 1985, A\&A, 144, 220

Aspin, C., \& Reipurth, B. 2000, MNRAS, 311, 522

Bally, J., \& Devine, D. 1994, ApJ, 428, L65

Bechis, K. P., Harvey, P. M., Campbell, M. F., \& Hoffmann, W. F. 1978, ApJ, 226, 439

Bertout, C. 1987, Circumstellar Matter, IAU Symp., 122, 23

Burton, M. G., \& Allen, D. A. 1994, Astronomy with Arrays, The Next Generation, ASSL, 190, 61

Cabrit, S., Lagage, P., McCaughrean, M., \& Olofsson, G. 1997, A\&A, 321,523

Calvet, N., \& Cohen, M. 1978, MNRAS, 182, 687

Canto, J., Rodriguez, L. F., Calvet, N., \& Levreault, R. M. 1984, ApJ, 282, 631

Cohen, M., Kuhi, L. V., Spinrad, H., \& Harlan, E. A. 1981, ApJ, 245, 920

Cohen, M., \& Schwartz, R. D. 1983, ApJ, 265, 877

Chavarria-K., C. 1985, A\&A, 148, 317

Corcoran, D., \& Ray, T. P. 1995, A\&A, 301, 729

Corcoran, M., \& Ray, T. P. 1997, A\&A, 321, 189

Corcoran, M., \& Ray, T. P. 1998, A\&A, 336, 535

Corcoran, D., Ray, T. P., \& Bastien, P. 1995, A\&A, 293, 550 (CRB)

Craine, E. R., Byard, P. L., \& Boeshaar, G. O. 1981, AJ, 86, 751

Downes, T. P., \& Cabrit, S. 2003, A\&A, 403, 135

Dent, W. R. F., \& Aspin, C. 1992, MNRAS, 259, 401

Edwards, S., \& Snell, R. L. 1983, ApJ, 270, 605

Eiroa, C., Gomez de Castro, A. I., \& Miranda, L. F. 1992, A\&AS, 92, 721

Eisloffel, J., \& Mundt, R. 1997, AJ, 114, 280

Eislöffel, J. 2000, A\&A, 354, 236

Fuente, A., Neri, R., Martín-Pintado, J., et al. 2001, A\&A, 366, 873

Goodrich, R. W. 1986, AJ, 92, 885

Goodrich, R. W. 1993, ApJS, 88, 609

Gomez, M., Kenyon, S. J., \& Whitney, B. A. 1997, AJ, 114, 265

Gómez de Castro, A. I., \& Robles, A. 1999, A\&A, 344, 632

Harvey, P. M., Wilking, B. A., \& Joy, M. 1984, ApJ, 278, 156

Harvey, P. M., Joy, M., Lester, D. F., \& Wilking, B. A. 1986, ApJ, 301, 346

Hartigan, P., Edwards, S., \& Ghandour, L. 1995, ApJ, 452, 736

Hartigan, P., Morse, J., \& Bally, J. 2000, AJ, 120, 1436

Heathcote, S., Morse, J. A., Hartigan, P., et al. 1996, AJ, 112, 1141

Herbig, G. H. 1960, ApJS, 4, 337

Hester, J. J., Stapelfeldt, K. R., \& Scowen, P. A. 1998, AJ, 116, 372

Hunter, T. R., Phillips, T. G., \& Menten, K. M. 1997, ApJ, 478, 283

Kwan, J., \& Tademaru, E. 1995, ApJ, 454, 382

Lagage, P. O., Olofsson, G., Cabrit, S., et al. 1993, ApJ, 417, L79

Leinert, C., Haas, M., \& Weitzel, N. 1993, A\&A, 271, 535

Marti, J., Rodriguez, L. F., \& Reipurth, B. 1993, ApJ, 416, 208

Miranda, L. F., Eiroa, C., \& Gomez de Castro, A. I. 1993, A\&A, 271, 564

Mitchell, G. F., \& Matthews, H. E. 1994, ApJ, 423, L55

Mundt, R., \& Ray, T. P. 1994, The Nature and Evolutionary Status of Herbig Ae/Be Stars, ASP Conf. Ser., 62, 237 
Mundt, R., Bührke, T., Fried, J. W., et al. 1984, A\&A, 140, 17 Mundt, R., Brugel, E. W., \& Bührke, T. 1987, ApJ, 319, 275 O’Dell, C. R., Hartigan, P., Lane, W. M., et al. 1997, AJ, 114, 730 Ogura, K. 1995, ApJ, 450, L23

Palla, F., \& Stahler, S. W. 1993, ApJ, 418, 414

Parthasarathy, M., Vijapurkar, J., \& Drilling, J. S. 2000, A\&AS, 145, 269

Phillips, J. P., \& Mampaso, A. 1996, A\&A, 316, 182

Ray, T. P. 1987 , A\&A, 171, 145

Ray, T. P., Poetzel, R., Solf, J., \& Mundt, R. 1990, ApJ, 357, L45

Reipurth, B. 1989, Nature, 340, 42

Reipurth, B., \& Bally, J. 2001, ARA\&A, 39, 403

Reipurth, B., Bally, J., \& Devine, D. 1997, AJ, 114, 2708

Reipurth, B., Chini, R., Krugel, E., Kreysa, E., \& Sievers, A. 1993, A\&A, 273, 221

Rodriguez, L. F., Moran, J. M., \& Ho, P. T. P. 1980, ApJ, 240, L149
Scarrott, S. M., Rolph, C. D., \& Tadhunter, C. N. 1991, MNRAS, 249, 310

Schwartz, R. D., Wilking, B. A., \& Giulbudagian, A. L. 1991, ApJ, 370, 263

Shepherd, D. S., Churchwell, E., \& Wilner, D. J. 1997, ApJ, 482, 355

Shepherd, D. S., Watson, A. M., Sargent, A. I., \& Churchwell, E. 1998, ApJ, 507, 861

Shevchenko, V. S., \& Yakubov, S. D. 1989, Sov. Astron., 33, 370

Staude, H. J., \& Elsasser, H. 1993, A\&ARv, 5, 165

Stone, J. M., Ostriker, E. C., \& Gammie, C. F. 1998, ApJ, 508, L99

Strom, K. M., Strom, S. E., Wenz, M., Wolff, S. C., \& Morgan, J. 1986, ApJS, 62, 39

Vilchez, J. M., Mampaso, A., Riera, A., \& Phillips, J. P. 1989, A\&A, 213, 303

Ward-Thompson, D. 2002, Science, 295, 76

Weintraub, D. A., Kastner, J. H., \& Mahesh, A. 1994, ApJ, 420, L87 